\title{
WLAN-BASED INDOOR LOCALIZATION USING NEURAL NETWORKS
}

\author{
Fasiha Saleem $^{* *}$ - Shurjeel Wyne*
}

\begin{abstract}
Wireless indoor localization has generated recent research interest due to its numerous applications. This work investigates Wi-Fi based indoor localization using two variants of the fingerprinting approach. Specifically, we study the application of an artificial neural network (ANN) for implementing the fingerprinting approach and compare its localization performance with a probabilistic fingerprinting method that is based on maximum likelihood estimation (MLE) of the user location. We incorporate spatial correlation of fading into our investigations, which is often neglected in simulation studies and leads to erroneous location estimates. The localization performance is quantified in terms of accuracy, precision, robustness, and complexity. Multiple methods for handling the case of missing APs in online stage are investigated. Our results indicate that ANN-based fingerprinting outperforms the probabilistic approach for all performance metrics considered in this work.
\end{abstract}

K e y w o r d s: wireless local area network, localization, channel fading, neural network

\section{INTRODUCTION}

A localization system determines the position of a person or object within a predefined coordinate system [1]. Indoor localization systems have received significant attention in the last few years because of many health and safety-related applications for locating patients, devices, or law-enforcement personnel in large buildings [2], [3]. The Global positioning system (GPS) signals are greatly attenuated while penetrating building materials [4], which necessitates the need for developing alternative indoor localization techniques. The initial systems developed for indoor localization have the drawback of requiring dedicated infrastructure and some specialized equipment to be worn by the person to be localized, see [5], [6] and [7]. However, in recent years the widespread availability of Wireless local area networks (WLANs) based on the IEEE802.11 standard, commonly termed as Wi$\mathrm{Fi}$, has led to new techniques for efficient indoor localization [8]. The common availability of multiple Wi-Fi access points (APs) in indoors and widespread use of portable smart devices allows cost-effective solutions for location estimation, which require minimal additional hardware; see for example [3], [7], [9], and [10]. The WLAN-based localization systems determine user location by utilizing the received signal strength (RSS), which is a measure of signal power received by a device, from multiple APs. The RSS values are measurable by a wireless network interface card even for private Wi-Fi networks that require a password for usage [4], [10].

The two major categories of WLAN-based localization techniques are Triangulation and Fingerprinting [1]. The triangulation approach infers target location by using the distance/angle information from at least three APs that act as reference locations. The required distance/angle information can be obtained by using the Time of arrival (TOA), Time difference of arrival (TDOA), or Angle of arrival (AOA) information of the signals received from the multiple APs [11], [12]. For indoor environments often the line-of-sight (LOS) path between the transmitter and receiver is not available due to severe attenuation by walls and other blocking objects. For this reason AOA, TDOA and TOA methods, which require a clear LOS path for operation, are not reliable methods for indoor localization [11].

In contrast, fingerprinting-based techniques use empirical data to estimate user location and are more robust to measurement errors relative to the triangulation techniques [7], [13]. A fingerprinting-based method operates in two stages: an offline stage in which the features/fingerprints from the region of interest are collected at some known locations named anchor locations, followed by an online stage in which the unknown location of the desired object/person is estimated by matching the online measurements with the closest a-priori location fingerprints. The fingerprinting approach provides accurate positioning even in complex indoor environments without the necessity to model the wireless propagation channel. In the literature, indoor localization has been investigated for many variants of the fingerprinting approach such as k-nearest-neighbor $(k-\mathrm{NN})$ [13], probabilistic methods [7] and artificial neural networks (ANNs) [3].

The $k$-NN approach offers a simple solution for estimating unknown user position by averaging the geographical coordinates of $\mathrm{k}$ closest reference points from the previously built database. However, this simplicity of implementation is acquired at the cost of relatively poor accuracy of location estimates [10]. Many authors have

\footnotetext{
${ }^{*}$ Department of Electrical Engineering, COMSATS Institute of Information Technology, Islamabad, Pakistan; ${ }^{* *}$ Department of Physics, COMSATS Institute of Information Technology, Islamabad, Pakistan, shurjeel.wyne@comsats.edu.pk
} 
also investigated the use of ANNs for indoor localization, see for example [14], [15], [16], [17], and [18]. In [14] the authors have used an ANN along with TOA and AOA methods to reduce location estimation errors in non-lineof-sight (NLOS) scenarios. In [15] the authors have compared a multilayer perceptron (MLP) ANN, combined with triangulation or trilateration techniques, with two variants of a Kalman filter. By experimental results, they have shown that MLP achieves a higher accuracy and requires lesser computational resources than the Kalman filter. In [16] the authors evaluated node localization in a wireless sensor network using an ANN and obtained higher accuracy than traditional schemes of location estimation. In [17], an adaptive neural fuzzy inference system was employed to model the dependency between RSS values and physical distances in the offline stage. In the online stage the distance between AP and target mobile device was determined by using this RSS-position dependency model and then an extended Kalman filter was used to compute the unknown position of mobile device in $2 \mathrm{D}$ space. It was concluded that the adaptive neural fuzzy system provides good distance estimates due to its nonlinear ability and fast learning capacity. However, the authors in [14], [15], [16], and [17] have not used an ANN in combination with the fingerprinting approach. A performance comparison of three types of dynamic neural networks was presented in [3]. It was shown that the feed forward time-delay neural network gives better location estimation with smaller training time than the nonlinear autoregressive network and the layer recurrent network. However, details of the chosen ANN parameters were not provided and the authors did not discuss how to handle the case of missing APs in the online phase; such a scenario may arise when RSS values from certain APs are available in the offline phase but are not received in online stage due to severe fading. In [18] an ANN with fingerprinting approach has been investigated and the authors propose a modular MLP-ANN approach to handle the problem of missing APs in online stage. The authors select an ANN architecture with two hidden layers that can result in increased complexity. All the aforementioned works have considered location independent shadow fading to simplify generation of theoretical RSS datasets for offline phase. However, neglecting the spatial correlation of the shadow fading can result in inaccurate performance analysis of localization algorithms [1].

This work revisits the localization performance of the modular MLP-ANN approach of [18], and also proposes alternative low-complexity approaches to handle missing APs in the online stage. In contrast to [18], our localization evaluations show that only one hidden layer in the ANN is sufficient to achieve good accuracy. We then compare the performance of the aforementioned ANNbased techniques with the probabilistic method, which is frequently used as a performance benchmark for localization evaluations [5], [19]. It may be noted that if interpolation is not used in the probabilistic method, the maximum likelihood estimate (MLE) of the unknown user location will always belong to the set of reference points measured in the offline phase. Therefore, we propose two simple interpolation methods for the MLE-based location fingerprinting. Our work incorporates spatial correlation effects of the shadow fading in the offline RSS database, which is generated from the model of [1].

\section{INDOOR POSITIONING WITH LOCATION FINGERPRINTING}

Indoor localization viewed in the context of an ANN is a function Artificial neural networks (ANNs) approximation problem, where the task is to approximate the nonlinear function that maps RSS values (inputs) to geographical coordinates (outputs) in the region of interest. A neural network is composed of multiple neurons arranged in a set of layers; each neuron in a given layer is connected to every neuron in the successive layer through adjustable weights that work together to approximate a function [20]. In its training/offline phase an ANN learns the non-linear function between observed RSS values (inputs) and the geographical locations (target outputs) of the measurement, by adjusting its weights to minimize the error between the ANNs training outputs and its target outputs [21]. Once the network has been trained, it can estimate the unknown location of user equipment (UE) in the online phase by using RSS measurements only.

In constructing the ANN architecture for indoor localization, we have adopted the approach of [2], [20], [21], [22] [23] to use a feed-forward multi-layer perceptron architecture because it presents a good tradeoff between accuracy and memory requirements; the latter is desirable in view of memory and computational constraints of many handheld smart devices. We consider a threelayered MLP architecture, ie, a single hidden layer in addition to the input and output layers. The choice of only a single hidden layer is motivated by the universal approximation theorem, which states that a single correctly dimensioned hidden layer is sufficient for approximating continuous non-linear functions [1], [20]. The number of nodes in the input layer equals the number of APs measured in the offline stage. In the output layer there are two neurons corresponding to two-dimensional (2D) coordinates of the unknown user location, ie, a 2D localization problem is considered herein. Finally, to dimension the hidden layer appropriately, one approach suggested in [22] is to select the number of hidden layer neurons such that the total number of weights in the network is approximately a tenth of the available training samples. However, many works have used more than this number of hidden neurons, see [22] and references therein. A more structured approach suggested by [22] is to initially start with a large number of hidden neurons then to reduce this number gradually while the estimation error is within acceptable limits. We have adopted the latter approach whereby the network was trained for over 100 epochs with an error of 10-6 being set as the training goal. After substantial trials we determined that 10 neurons in 
the hidden layer were sufficient to provide the desired accuracy within the given training restrictions.

In selecting an activation function for the hidden layer neurons Sigmoid functions are widely used because hidden neurons with sigmoid function are responsive for a broad range of inputs, i.e., it is more likely that a given input value influences several hidden neurons [22]. We have chosen a hyperbolic tangent sigmoid function for the hidden layer neurons of the considered ANN. Furthermore, for the output layer neurons a linear activation function is used for function approximation problems because in this case the output values can take values in a continuous range rather than being divided into classes. To facilitate an appropriate selection of the network training function for an ANN, the authors in [24] present an empirical investigation of different network training functions for various benchmark problems; for function approximation problems the Levenberg-Marquardt training algorithm is suggested because it produces lower error than other training functions. Furthermore, the Levenberg-Marquardt training algorithm has also been demonstrated to have the fastest convergence rate for networks having less than a few hundred weights [24]. In view of these considerations, we have selected the Levenberg-Marquardt training algorithm for the ANN considered in this work.

\subsection{Probabilistic methods}

Probabilistic fingerprinting techniques treat RSS values at a location as random variables and utilize the statistical distribution of the RSS values to identify an unknown location [25]. Let $l$ denote a location and o the vector of RSS values received at this location from all available APs. Assuming there are $n$ anchor point locations and RSS values from available APs are collected at each location in the offline phase. Then in the online phase, the user at an unknown location will measure a vector of RSS values and according to the Probabilistic fingerprinting method, the anchor point location that has the highest posteriori distribution $p\left(l_{i} / \mathbf{o}\right)$ will be returned as an estimate of the unknown user location [23] [26].

Mathematically: Select $l_{i}$ if $p\left(l_{i} / \mathbf{o}\right)>p\left(l_{j} / \mathbf{o}\right), i, j=$ $1,2,3, \ldots, n, j \neq i$.

According to the well-known Bayes rule

$$
p\left(\frac{l_{i}}{\mathbf{o}}\right)=\frac{p\left(\frac{\mathbf{o}}{l_{i}}\right) p\left(l_{i}\right)}{p(\mathbf{o})}
$$

where $p\left(l_{i}\right)$ is the prior probability of location $l_{i}$ before receiving o. Assuming that the anchor locations have an identical probability of being chosen, $p\left(l_{i}\right)$ will be a uniform distribution that is identical for all locations. Furthermore, $p(\mathbf{o})$ given by,

$$
p(\mathbf{o})=\sum_{i=1}^{n} p\left(\frac{\mathbf{o}}{l_{i}}\right) p\left(l_{i}\right),
$$

is not a function of location and is therefore identical for all locations $l_{i}$ in the region of interest. The term $p\left(\mathbf{o} / l_{i}\right)$ seen in (1) is the likelihood function of location $l_{i}$, which determines the probability of observing RSS vector o at location $l_{i}$. Therefore, to determine the highest posteriori probability we require to maximize $p\left(\mathbf{o} / l_{i}\right)$ only while treating $p(\mathbf{o})$ and $p\left(l_{i}\right)$ as constants. Now considering a location $l_{i}$, the likelihood function at $l_{i}$ is estimated as the product of likelihood functions for all $m$ available APs as,

$$
p\left(\frac{\mathbf{o}}{l_{i}}\right)=\prod_{j=1}^{m} p\left(\frac{o_{j}}{l_{i}}\right),
$$

where $o_{j}$ is the RSS received from $j$-th AP at location $l_{i}$. This method of approximating user location using a probabilistic approach is known as maximum likelihood estimation (MLE) [19]. One drawback of this approach is that it can only estimate the unknown user location as one of the anchor point locations. For more accurate estimation of the unknown location, interpolation between the anchor locations can be introduced. Two different ways of interpolating the locations have been proposed in the literature.

\section{MLE with interpolation method 1}

The coordinates of the unknown UE location can be estimated as a weighted sum of the coordinates of all anchor locations with the posteriori probabilities of these locations adopted as their respective weights. The estimated location coordinates of UE are then expressed as [23].

$$
(\hat{x}, \hat{y})=\sum_{i=1}^{n} p\left(\frac{l_{i}}{\mathbf{o}}\right) \cdot\left(x_{l_{i}}, y_{l_{i}}\right)
$$

\section{MLE with interpolation method 2}

In this approach, anchor locations are sorted according to their descending posteriori probability values and the $\mathrm{k}$ largest posteriori probability location coordinates are averaged to obtain an estimate of the unknown location coordinates as $[5]$,

$$
(\hat{x}, \hat{y})=\frac{1}{k} \sum_{i=1}^{k}\left(x_{l_{i}}, y_{l_{i}}\right)
$$

where $k$ is taken to be the number of APs available in the scenario under consideration [5].

\section{SIMULATION MODEL}

For evaluating the localization performance of the selected techniques, we adopt the indoor scenario proposed in [27] which consists of a room of size $20 \times 20 \mathrm{~m}^{2}$ as shown in Fig. 1. There are four APs deployed at four corners of the room and 25 anchor locations are equally spaced on a grid with a spacing of $4 \mathrm{~m}$ between adjacent anchors. All simulation results have been generated by using the MATLAB computational software. 


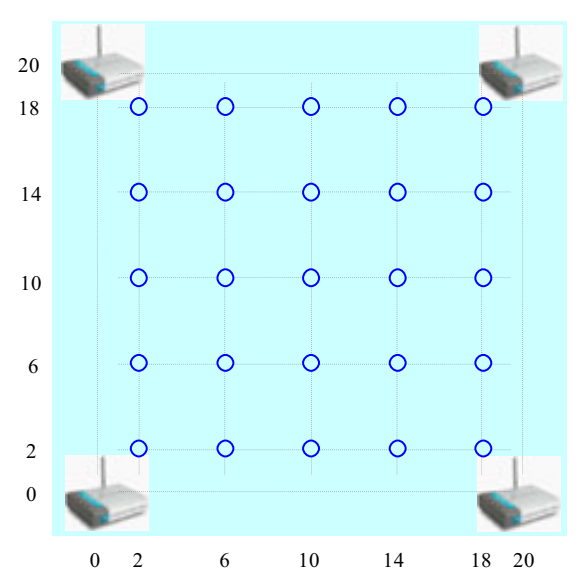

Fig. 1. Simulation layout for room size $20 \times 20 \mathrm{~m}^{2}$ with 4 APs in corners and 25 anchor locations, [27]

Table 1. Parameters of Propagation model, [28]

\begin{tabular}{cccccc}
\hline Parameter & $d_{0}$ & $P_{\mathrm{r}}\left(d_{0}\right)$ & $n$ & $\sigma$ & $D_{\mathrm{c}}$ \\
& $(\mathrm{m})$ & $(\mathrm{dBm})$ & $(-)$ & $(\mathrm{dB})$ & $(\mathrm{m})$ \\
\hline Value & 1 & -40 & 2.0 & 3.0 & 10 \\
\hline
\end{tabular}

The RSS value at distance $d$ from an AP is computed according to the propagation model [27],

$$
P_{r}(d)=P_{r}\left(d_{0}\right)-10 n \log \left(\frac{d}{d_{0}}\right)-X_{\sigma} .
$$

Here $d_{0}$ is the reference distance, $P_{r}(d)$ and $P_{r}\left(d_{0}\right)$ denote the received signal power at distance $d$ and $d_{0}$, respectively $[27,28]$. Further, $n$ is the path loss exponent and $X_{\sigma}$ is a zero mean Gaussian random variable with standard deviation $\sigma$, which models the shadow fading due to signal blockage in the indoor scenario. A spatial correlation is induced into the shadow fading observed at close-in physical locations due to the similarity in environmental obstructions encountered in the signal propagation path [1]. Therefore, accurate evaluation of the localization performance requires that this correlation is also taken into consideration when generating the RSS dataset [1]. The parameter values for the propagation model in (6) have been selected after careful considerations of the indoor environment $[27,28]$ and are shown in Table 1. For computing spatially correlated shadow fading values, a covariance matrix $\mathbf{K}$ is generated whose element $K_{i j}$ is obtained as [1]

$$
K_{i j}\left(d_{i j}\right)=\sigma^{2} \exp \left(-\frac{d_{i j}}{D_{c}} \ln 2\right)
$$

where $D_{c}$ is the correlation distance $\mathbf{K}$ is decomposed as $\mathbf{K}=\mathbf{L L}^{\top}$ [29] and then matrix $\mathbf{L}$ is used to generate correlated shadow fading values in the vector $\mathbf{X}_{\sigma}$ as,

$$
\mathbf{X}_{\sigma}=\mathbf{L w}
$$

where $\mathbf{w}$ is a vector of uncorrelated random variables with zero mean and unit variance. The selected values for $D_{c}$ and $\sigma$ are shown in Table 1 for the considered indoor environment.

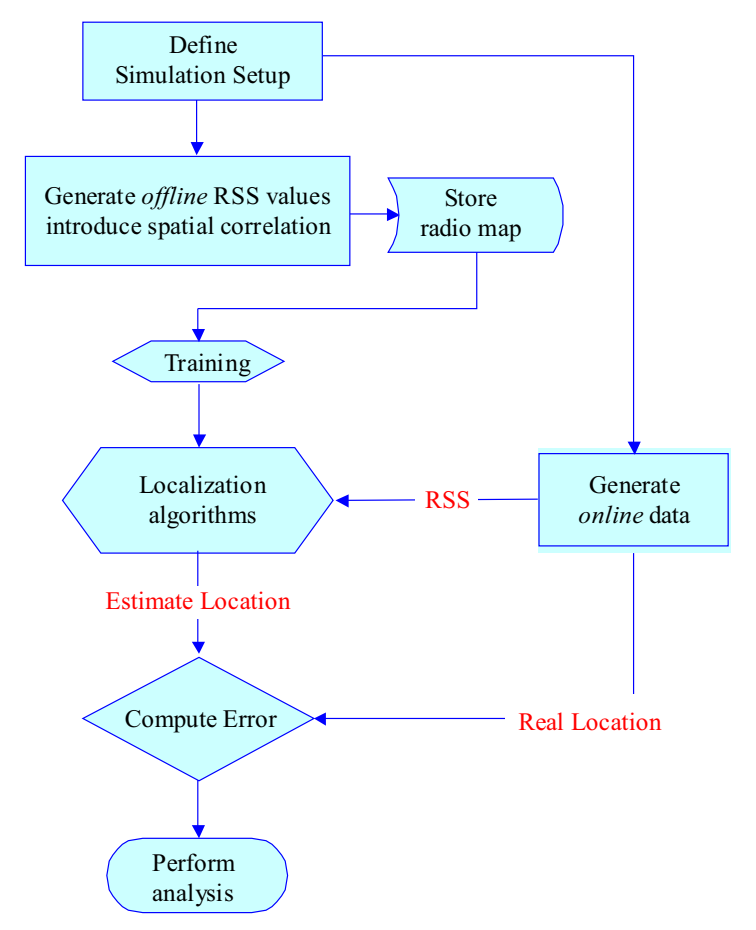

Fig. 2. Block diagram of the simulation procedure

The complete simulation procedure is illustrated by the flowchart given in Fig. 2, the RSS values from all available APs are computed at each anchor location in the offline phase. This dataset is used for training the ANN and also for acquiring the offline dataset for the probabilistic methods, ie, mean and standard deviation of RSS values. For the online phase, a single trial is executed by randomly placing the UE in the room according to a uniform distribution of its $x$ and $y$ coordinates; thus any position within the room is equally likely for the online phase, which constitutes the worst case scenario from an estimation perspective. The RSS values from the available APs in online phase are then generated according to the model of (6). The UE location estimated by the localization algorithm was compared with the true location to compute the localization error for a single trial. In this way 10000 independent trials were conducted to collect error statistics for the reported performance evaluation.

\section{PERFORMANCE METRICS AND RESULTS}

The performance of a localization technique is typically quantified by metrics such as localization accuracy, precision, robustness and computational complexity [23]. These metrics are used in the sequel for performance comparison of the considered ANN and probabilistic methods. 


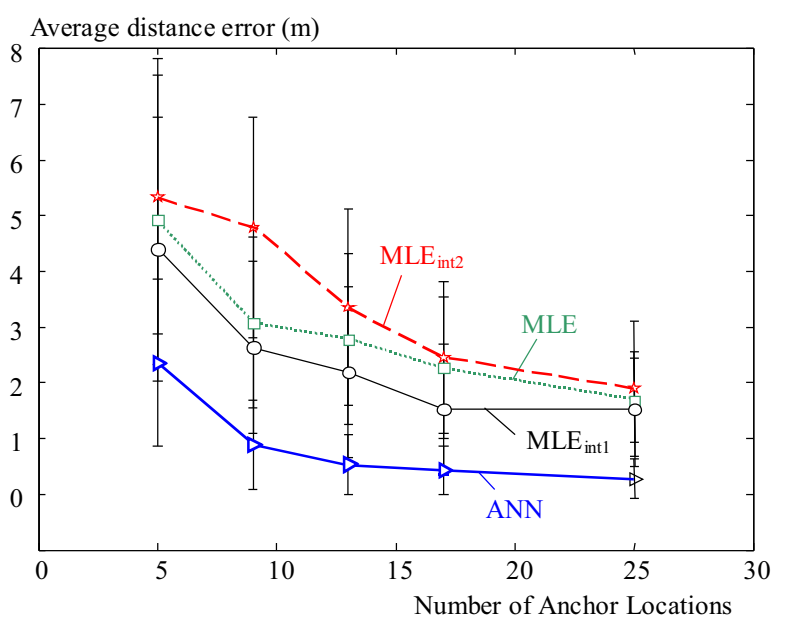

Fig. 3. Accuracy of ANN and probabilistic methods

\section{Accuracy}

The accuracy is measured by the mean value of the distance error which is computed as [23],

$$
\bar{E}_{d}=\frac{1}{N} \sum_{i=1}^{N} E_{d}
$$

where $N$ is the number of independent trials and $E_{d}$ is the Euclidean distance between the estimated location and the true location of UE. For the 2D localization problem considered herein, the Euclidean distance is written as,

$$
E_{d}=\sqrt{(x-\hat{x})^{2}+(y-\hat{y})^{2}}
$$

where, $(x, y)$ and $(\hat{x}, \hat{y})$ are the $x$ and $y$ location coordinates of the true and estimated locations, respectively. A smaller mean error in the location estimate is desirable and leads to a more accurate localization algorithm. In Fig. 3, the mean distance error and the error deviation about the mean for ANN and the three probabilistic methods are plotted against the number of anchor points. From the figure it can be seen that by increasing the number of anchor locations in offline phase, both the average distance error as well as the error deviation about the mean decrease monotonically for all localization algorithms. However, the mean and standard deviation of the location estimation error for ANN is smaller than all three variants of the MLE method. With 5 anchor locations, average error and its deviation are $2.35 \mathrm{~m}$ and 1.50 $\mathrm{m}$ for ANN and $4.40 \mathrm{~m}$ and $2.38 \mathrm{~m}$ for MLE with interpolation method 1. With 13 anchor locations, average error and its deviation are $0.53 \mathrm{~m}$ and $0.52 \mathrm{~m}$ for ANN and $2.16 \mathrm{~m}$ and $1.54 \mathrm{~m}$ for MLE with interpolation method 1. With 25 anchor locations, average error and its deviation are $0.27 \mathrm{~m}$ and $0.36 \mathrm{~m}$ for ANN and $1.54 \mathrm{~m}$ and 1.03 $\mathrm{m}$ for MLE with interpolation method 1. It can be seen from the figure that MLE in conjunction with interpolation method 1 yields the smallest average error of 1.54 $\mathrm{m}$ among the probabilistic methods while the other two probabilistic methods yield $1.69 \mathrm{~m}$ and $1.90 \mathrm{~m}$ average error values.

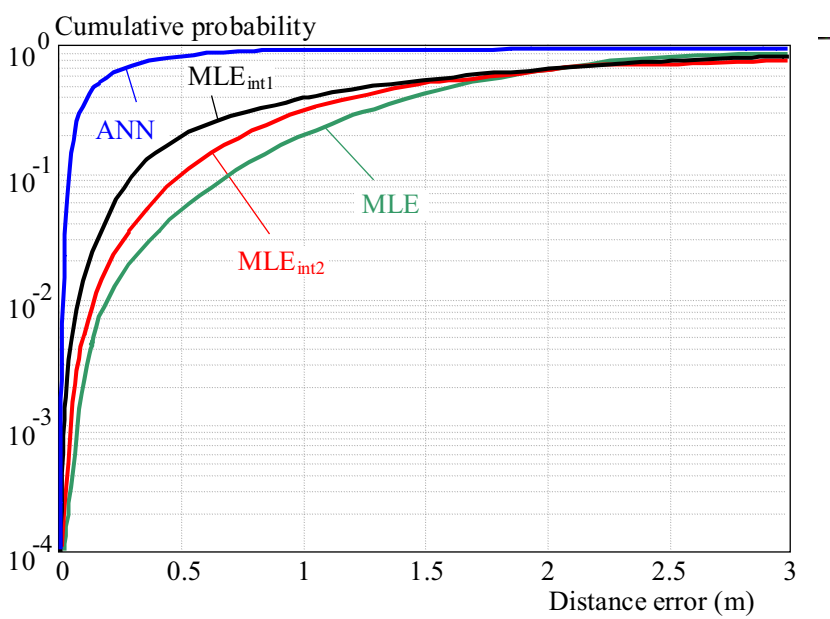

Fig. 4. CDF of localization error for $\mathrm{ANN}$ and probabilistic approaches

\section{Precision}

While accuracy considers the average error, precision explores the distribution of error. A localization algorithm that exhibits an error distribution in which small errors occur with higher probability and large errors occur with lower probabilities is preferred [11] [23]. We have used the cumulative distribution function (CDF) of error distance for investigating the precision of the localization algorithms [11]. The CDF plot of error for ANN and three probabilistic approaches is shown in Fig. 4 for 25 anchor locations. From Fig. 4 it is clear that ANN has higher probability for low errors. For example, percentage probability of achieving less than or equal to $0.5 \mathrm{~m}$ error is $86 \%$ for ANN whereas this probability is only $10 \%, 19.5 \%$ and $5 \%$ for the three probabilistic methods. If we make a comparison among probabilistic approaches, it can be seen that MLE with interpolation method 1 returns a distance error below $0.5 \mathrm{~m}$ with probability $19.5 \%$, which is significantly larger than the other two interpolation methods, which have only $10 \%$ and $5 \%$ probability of giving error values below $0.5 \mathrm{~m}$, ie, higher distance errors are more probable with these approaches relative to MLE with interpolation method 1 . In view of the better accuracy and precision performance of the MLE with interpolation method 1 relative to other two MLE variants, we consider only the MLE with interpolation method 1 for subsequent evaluations of algorithm robustness and complexity in comparison with the ANN-based approach.

\section{Robustness}

Robustness of a localization technique is a measure of its ability to provide reasonable location estimates in the absence of RSS values from one or more APs in the online phase. Such a scenario may arise if the signals from certain APs, that were available during training phase, are significantly attenuated due to severe channel fading in the online phase. For investigating the algorithm robustness to missing RSS values, test data for online phase is generated in which RSS values from AP1 shown in Fig. 1 are not available while valid RSS values from 
remaining three APs are received. The error statistics for both the ANN and MLE with interpolation method 1 are then evaluated for this scenario.

For the MLE method, a solution for handling the missing AP is to set likelihood $p\left(\mathbf{o}_{j} / l\right)=1$ at all locations for $j$-th missing AP [23]. For the ANN approach, multiple ways of handling this situation are investigated as follows. One simple approach is to replace the missing RSS value in the online phase by a suitable value. We propose three possibilities of choosing this value as described below:

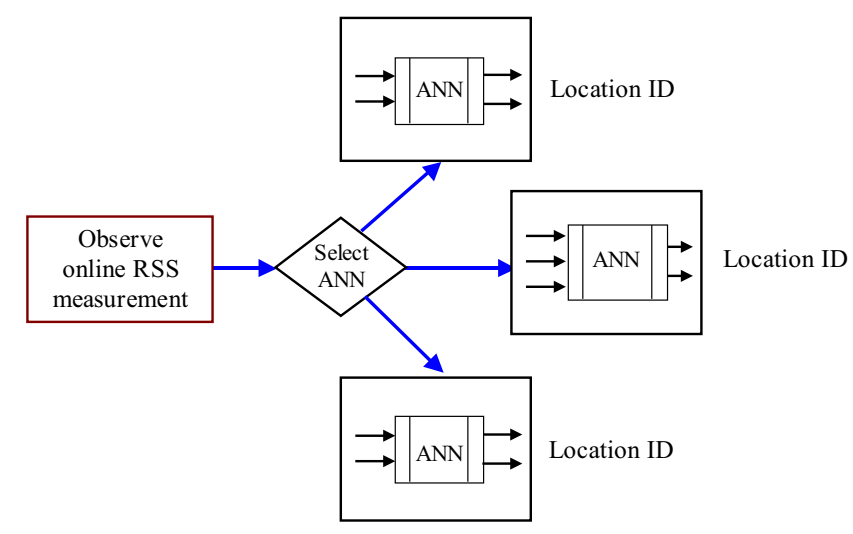

Fig. 5. Modular ANN selection scheme to handle missing APs in online stage, [18]

\section{Mean 1:}

Replace the missing RSS value with the mean of offline phase RSS values of missing AP at all anchor locations as

$$
\text { Mean } 1=\frac{1}{n} \sum_{i=1}^{n} \operatorname{RSS}_{i}
$$

where $\operatorname{RSS} j_{i}$ is the RSS value from $j$-th AP at $i$-th location in the offline phase $(i=1,2, n)$, and RSS values from the $j$-th AP are not available in the online phase.

\section{Mean 2:}

Select the RSS value from the missing AP to be the RSS value that was available in training phase at an anchor location centrally located in the considered region of interest; for example, the missing online RSS value is replaced by its offline value at the central location in the considered simulation setup with coordinates $(x, y)$ $=(10,10), i e$, the missing $\mathrm{RSS}$ value is given as,

$$
\text { Mean } 2=\operatorname{RSS} j_{(10,10)}
$$

\section{Mean 3:}

RSS value of $j$-th AP, which is missing in online phase, is replaced with mean of RSS values of remaining APs, which are available in the online phase, ie, the missing RSS value is obtained as,

$$
\text { Mean } 3=\frac{1}{m-1} \sum_{i=1, i \neq j}^{m} \operatorname{RSS}_{i}
$$

where, $\mathrm{RSS}_{i}$ is the RSS value from $i$-th AP that is available in the online phase.

In [18] an alternative approach to handle the problem of missing AP has been suggested whereby different neural networks are trained in offline phase by considering the absence of RSS values from a particular AP. Then, in the online phase, the appropriate ANN is selected according to the available set of RSS values as shown in Fig. 5, [18].

Figure 6 plots the distribution of location errors for 25 anchor locations for the four methods discussed above. Here $\mathrm{ANN}_{3}$ denotes a stored ANN with 3 inputs, ie, one input corresponding to the missing AP has been discarded from the original set of four inputs. Furthermore, ANNm1, ANNm2 and ANNm3 denote the ANN-based approach with the missing RSS value replaced by one of the three means discussed above. It can be observed from Fig. 6 that ANN with 3 inputs (ANN3) is better than all other techniques. Furthermore, it is evident that even the ANN approach that uses one of the three means to replace the missing RSS is more robust than MLE with interpolation method 1. Probability of obtaining low error $e g 0.5 \mathrm{~m}$ is $51 \%, 11 \%, 10.5 \%, 9 \%$ and $3.8 \%$ for ANN3, ANNm1, ANNm2, ANNm3, and MLE with interpolation method 1 , respectively.

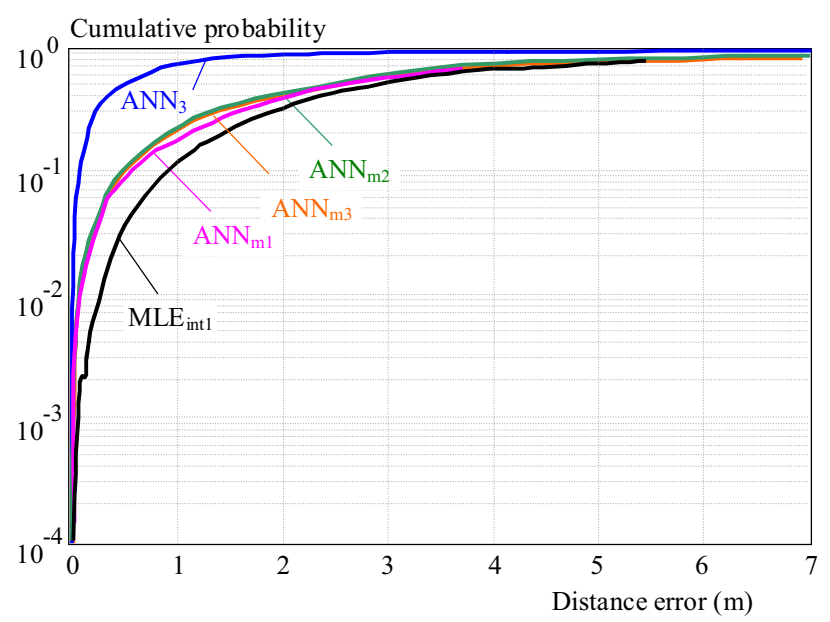

Fig. 6. Error CDF for different ANN schemes and MLE with interpolation method $1, \mathrm{AP} 1$ is assumed to be missing in the online stage

The comparison of the localization error of ANN with 3 inputs (ANN3), MLE with interpolation method 1, and ANN with mean 1 is demonstrated in Fig. 7 for different number of anchor points. It can be seen that average distance error as well as the error deviation decreases with increasing number of anchor locations. However, the mean and standard deviation of the localization error of ANN3 method is lower than the other approaches. For example, from Fig. 7 it can be seen that for 25 anchor locations, ANN3 method has an average error and deviation of $0.84 \mathrm{~m}$ and $1.24 \mathrm{~m}$, respectively. While for ANNm1 method the average error and deviation are $2.90 \mathrm{~m}$ and 
$2.45 \mathrm{~m}$ respectively. Finally for MLE with interpolation method 1 , the average error and deviation are $3.34 \mathrm{~m}$ and $2.45 \mathrm{~m}$, respectively. Hence it can be concluded that the ANN with 3 inputs has the most robust localization performance against missing RSS values. However, the ANN using the mean is more robust than MLE with interpolation method 1 .

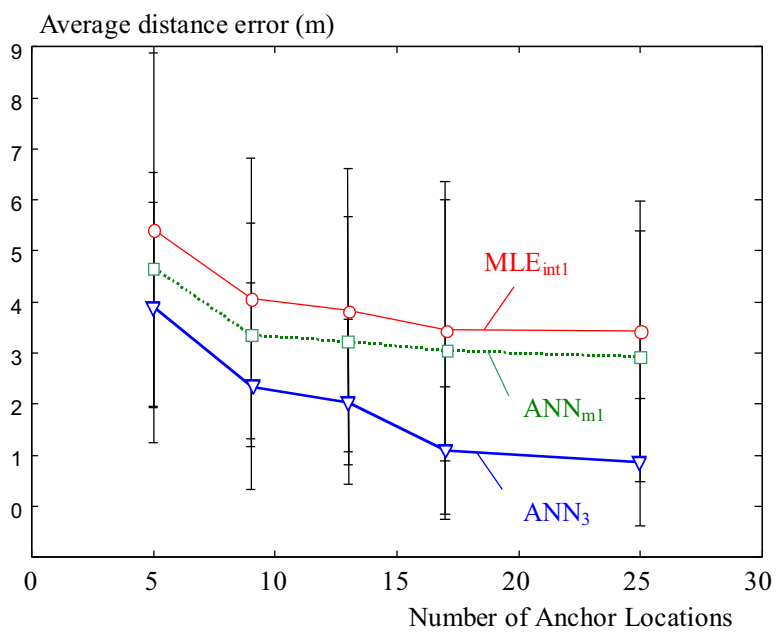

Fig. 7. Accuracy of ANN schemes and MLE with interpolation method 1, AP1 is assumed to be missing in the online stage

\section{Complexity}

A localization algorithm that provides the desired accuracy with minimal computational complexity is desirable. We follow the approach of [23] in using the processing time of an algorithm, which is required to estimate the unknown UE location, as a measure of the algorithm computational complexity. The average runtime over 20 independent trials for each algorithm was evaluated in MATLAB version 8.1.0.604 (R2013a) on a desktop machine hp CORE i3 with $4.00 \mathrm{~GB}$ RAM and a $2.4 \mathrm{GHz}$ processor. The emphasis here was to obtain a measure of relative computational complexity of the algorithms rather than an absolute measure of computational time. The processing time was measured separately for the offline and online phases for ANN and MLE with interpolation method 1 and the results are provided in Table 2 .

Table 2. Processing time of localization algorithms in seconds

\begin{tabular}{cccc}
\hline \multicolumn{2}{c}{ Offline-phase } & \multicolumn{2}{r}{ Online-phase } \\
ANN & $M L E \_i n t 1$ & ANN & MLE_int 1 \\
\hline 1.0526654 & 0.011679 & 0.00000784 & 0.0040 \\
\hline
\end{tabular}

From Table 2 it can be seen that the ANN approach requires two orders of magnitude larger computational time than MLE with interpolation method 1 in the offline phase. This is because the ANN requires training during which its weights are adjusted. However, in the online phase, the processing time of ANN is three orders of magnitude smaller than the processing time required by MLE with interpolation method 1. Although the offline complexity for ANN is larger, this phase is encountered only once when the ANN is initially trained. In contrast, the processing time of the online phase is more critical because the online phase is invoked each time the UE location has to be estimated. With these considerations, the ANN algorithm that requires lesser processing time for online phase is preferable.

\section{CONCLUSION AND FUTURE WORK}

We have compared the indoor localization performance of an ANN with the MLE-based fingerprinting approach by using RSS values from multiple APs of a WLAN. For the MLE-based approach, two different interpolation methods have been considered in addition to the basic MLE localization without interpolation. Our evaluations conclude that ANN-based localization outperforms all variants of the MLE approach for the performance metrics considered in this work, whereas among the MLE variants the interpolation method 1 outperforms the other two MLE approaches. The mean localization error and the error deviation about the mean for the ANNbased approach are consistently smaller than those of the MLE with interpolation method 1 under identical conditions. For example, with 25 anchor locations the mean error and error deviation for the ANN approach are 0.27 $\mathrm{m}$ and $0.36 \mathrm{~m}$, respectively. While under the same conditions the mean error and error deviation for MLE with interpolation method 1 are $1.54 \mathrm{~m}$ and $1.03 \mathrm{~m}$, respectively. On the other hand, the average error for MLE with interpolation method 2 is $1.9 \mathrm{~m}$ and for MLE without interpolation the mean error is $1.69 \mathrm{~m}$. The probability of estimating the true user location within an error of 0.5 $\mathrm{m}$ is $86 \%$ for the ANN whereas the probability of the same event is $19.5 \%$ for MLE with interpolation method 1, $10 \%$ for MLE with interpolation method 2, and only $5 \%$ for the basic MLE localization without interpolation. To evaluate the robustness of localization performance in the event of a missing AP, four different variants of the ANN-based localization were investigated and compared with MLE with interpolation method 1. The modular ANN approach, which stores network parameters of multiple ANNs, each trained with a different subset of the set of all APs, was shown to outperform the other three variants of the ANN approach. However, all ANN variants outperform the localization performance of MLE with interpolation with method 1 . For example, the probability of estimating the true user location within an error of 0.5 $\mathrm{m}$ is $51 \%$ for ANN3, $11 \%$, for ANNm1, $10.5 \%$ for ANNm2, $9 \%$ for ANNm3 and only $3.8 \%$ for MLE with interpolation method 1. Future work will focus on investigating localization performance of the considered methods with measured RSS values in practical indoor scenarios. 


\section{Acknowledgment}

The authors wish to acknowledge partial funding of this work by the EU ATOM-690750 Research Project, approved under the call H2020-MSCA-RISE-2015.

\section{REFERENCES}

[1] SEYED, A.-ZEKAVAT, R-BUEHRER, R. M. : Handbook of Position Location: Theory, Practice and Advances, Wiley-IEEE Press, 2011.

[2] STELlA, M-RUSSO, M.-BEGUI, D. : F localization in indoor environment, Radioengineering 21 No. 2 (2012), 557-567.

[3] DJABRI, F.-LIU, R.: Wi-Fi-Based localization in dynamic indoor environment using a dynamic neural network, International Journal of Machine Learning and Computing 3 No. 1 (2013), 127-131.

[4] ALONSO, J. M. et al: Towards People Indoor Localization Combining WiFi and Human Motion Recognition, XV Congreso Espanol Sobre Tecnologas y Lgica Fuzzy (2010), 7-12.

[5] DAWES, B.-CHIN, K.-W.: A comparison of deterministic and probabilistic methods for indoor localization, The Journal of Systems and Software 84 No. 3 (2011), 442-451.

[6] DEASY, T. P.-SCANLON, W. G. : Stepwise algorithms for improving the accuracy of both deterministic and probabilistic methods in WLAN-based indoor user localization, International Journal of Wireless Information Networks 11 No. 4 (2005), 207-216.

[7] YIM, J.: Introducing a decision tree-based indoor positioning technique, Expert Systems with Applications 34 No. 2 (2008), 1296-1302.

[8] CHEN, Q.-LEE, D.-L.-LEE, W.-CH. : Rule-based WiFi localization methods, IEEE/IFIP International Conference on Embedded and Ubiquitous Computing (EUC) (2008), 252-258.

[9] MENGUAL, L.-MARBÁN, O.-EIBE, S. : Clustering-based location in wireless networks, Expert Systems with Applications 37 No. 9, 6165-6175, 2010.

[10] FENG, CH.-AU-W. S. A.-VALAEE-S.-TAN, Z. : Compressive sensing based positioning using RSS of WLAN access points, IEEE Proceedings of INFOCOM, 2010, 1-9.

[11] LIU, H.-DARABI, H.-BANERJEE, P-LIU, J. : Survey of wireless indoor positioning techniques and systems, IEEE Transactions on Systems, Man, and Cybernetics, Part C: Applications and Reviews 37 No. 6, 1067-1080, 2007.

[12] COMSA, C.-R.-LUO, J.-HAIMOVICHL, A.-SCHWARTZ, S. : Wireless localization using time difference of arrival in narrow-band multipath systems, in IEEE International Symposium on Signals, Circuits and Systems (ISSCS), 2007, 1-4.

[13] JEKABSONS, G.-KAIRISH, V.-ZURAVLYOV, V.: An Analysis of Wi-Fi Based Indoor Positioning Accuracy, Scientific Journal of Riga Technical University, Computer Sciences 47 No. 1, 131-137, 2011.

[14] CHEN, CH.-SH.: Artificial Neural Network for Location Estimation in Wireless Communication Systems, Sensor 12 No. 3, $27982817,2012$.

[15] SHAREEF, A.-ZHU, Y.-MUSAVI, M.-SHEN, B. : Comparison of MLP Neural Network and Kalman filter for Localization in wireless sensor netwoks, 19th IASTED International Conference on Parallel and Distributed Computing and Systems (2007), 323-330.

[16] TIAN, J.-P.-SHI, H.-CH.: Study of localization scheme base on neural network for wireless sensor networks, IET Conference on Wireless, Mobile and Sensor Networks (CCWMSN07) (2007), 64-67.

[17] WU, B.-F.-JEN, CH.-L.-CHANG, K.-CH.: Neural fuzzy based indoor localization by extending Kalman filtering with propagation channel modeling Kalman Filter, INTECH Open Access Publisher (2010).

[18] AHMAD, UY., : In-building localization using neural networks jour IEEE International Conference on Engineering of Intelligent Systems.

[19] FANG, S.-H.-LIN, T.-N.: Indoor location system based on discriminant-adaptive neural network in IEEE 802.11 environments, IEEE Transactions on Neural Networks 19 No. 11 (2008), 1973-1978.

[20] RAHMAN, M. S.-PARK, Y.-KIM, K.-D. : Localization of wireless sensor network using artificial neural network, IEEE 9th International Symposium on Communications and Information Technology (ISCIT) (2009), 639-642.

[21] SHAREEF, A.-ZHU, Y.-MUSAVI, M.: Localization using neural networks in wireless sensor networks, in Proceedings of the 1st International Conference on MOBILe Wireless MiddleWARE, Operating Systems, and Applications (2008), 639-642.

[22] DUDA, R. O.-HART, P. E.-STORK, D. G. : Pattern classification, John Wiley and Sons, 2000.

23] LIN, T.-N.-LIN, P.-CH.: Performance comparison of indoor positioning techniques based on location fingerprinting in wireless networks, International Conference on Wireless Networks, Communications and Mobile Computing (2005), 1569-1574.

[24] Online www.mathworks.com/help/nnet.

[25] HERRANZ, F.-HERNANDEZ, N.-OCANA, M.-BERGASA L. M.-ALONSO, D. : Intelligent Techniques applied to WiFi Localization Systems, Signal Journal 50 No. 45 (2009), 221-226.

26] ROOS, T.-MYLLYMAKI, P.-TIRRI, H.-MISIKANGAS, P.-SIEVANEN, J.: A probabilistic approach to WLAN user location estimation, International Journal of Wireless Information Networks 9 No. 3 (2002), 155-164.

[27] RAHMAN, M. S.-PARK, Y.-KIM, K.-D. : RSS-based indoor localization algorithm for wireless sensor network using generalized regression neural network, Arabian Journal for Science and Engineering 37 No. 4 (2012), 1043-1053.

[28] YANG, J.-CHEN, Y.: Indoor localization using improved RSS-based lateration methods, IEEE Global Telecommunications Conference (GLOBECOM) (2009), 4506-4511.

[29] LEON-GARCIA, A.- : Probability, Statistics and Random Processes for Electrical Engineering, Pearson/Prentice Hall, 2008.

Received 23 November 2015

Fasiha Saleem was born in Pakistan in 1988. She received the BSc, Eng (Hons) degree in Electrical Engineering in 2011 from University of Engineering and Technology, Taxila, Pakistan and MS degree in Electrical Engineering in 2015 from COMSATS Institute of Information Technology (CIIT), Islamabad, Pakistan. She is currently serving as Research Associate at CIIT. Her research interests include wireless communications, digital signal processing, and distributed computing.

Shurjeel Wyne received his $\mathrm{PhD}$ from Lund University, Sweden in March 2009. Between April 2009 and April 2010, he was a Post-Doctoral Research Fellow funded by the HighSpeed Wireless Centre at Lund University. Since June 2010 he is with the Department of Electrical Engineering at COMSATS Institute of Information Technology, Islamabad, Pakistan, where he is an Associate Professor. Dr Wynes research interests are in the area of wireless communications, particularly channel measurements and modeling, $60 \mathrm{GHz}$ Communications, relay networks, and Multiple-input Multiple-output (MIMO) systems. He is a Co-recipient of the best paper award of the Antennas and Propagation Track in IEEE VTC2013Spring and a senior member of the Institute of Electrical and Electronics Engineers (IEEE), USA. 